\title{
Density correlations in ultracold atomic Fermi gases
}

\author{
W. Belzig \\ University of Konstanz, Department of Physics, D-78457 Konstanz, Germany \\ C. Schroll and C. Bruder \\ Department of Physics and Astronomy, University of Basel, Klingelbergstrasse 82, 4056 Basel, Switzerland
}

(Received 15 March 2007; published 12 June 2007)

\begin{abstract}
We investigate density fluctuations in a coherent ensemble of interacting fermionic atoms. Adapting the concept of full counting statistics, well known from quantum optics and mesoscopic electron transport, we study second-order as well as higher-order correlators of density fluctuations. Using the mean-field BCS state to describe the whole interval between the BCS limit and the Bose-Einstein condensate (BEC) limit, we obtain an exact expression for the cumulant-generating function of the density fluctuations of an atomic cloud. In the two-dimensional case, we obtain a closed analytical expression. Poissonian fluctuations of a molecular condensate on the BEC side are strongly suppressed on the BCS side. The size of the fluctuations in the BCS limit is a direct measure of the pairing potential. We also discuss the BEC-BCS crossover of the third cumulant and the temperature dependence of the second cumulant.
\end{abstract}

DOI: $10.1103 /$ PhysRevA.75.063611

PACS number(s): 03.75.Ss, 03.75.Hh, 05.30.Fk

\section{INTRODUCTION}

Following the successful creation of Bose-Einstein condensates (BECs) in ultracold atomic clouds [1], recently ultracold fermionic clouds have been produced [2-6]. This has attracted a lot of attention both theoretically and experimentally, especially due to the ability to tune the mutual interaction between atoms via a Fano-Feshbach resonance. The unique opportunity to study the crossover from weak attractive to strong attractive interactions in one and the same system makes this interesting from a fundamental many-body point of view.

Theoretically, fermionic systems with weak attractive interaction are superfluids and as such described by the Bardeen-Cooper-Schrieffer (BCS) theory [7]. This theory can also describe the limit of stronger attractive interaction [8-10], in which a BEC of molecules is formed. In the crossover regime, the long-range nature of the interaction makes the BCS theory less accurate [11].

Recently, several experiments studied the strongly interacting BEC-BCS crossover regime using spin mixtures of ultracold fermionic gases (see [12] for a recent review). Measurements of the interaction energy of an ultracold fermionic gas near a Feshbach resonance were made, studying the impact of the interaction on the time-of-flight expansion [13]. Experimental investigation of collective excitations showed a strong dependence on the coupling strength $[14,15]$. Moreover, the condensation $[16,17]$ and the spatial correlations [18] of the fermionic atom pairs were observed in the full crossover regime. The pairing gap was measured directly via a spectroscopic technique in the whole crossover region [19]. Remarkably, the gap values are in relatively good agreement with the BCS model in the whole region.

The use of noise correlations to probe the many-body states of ultracold atoms was proposed in [20] (see also [21]). Correlation measurements can be applied to detect phase coherence in mesoscopic superpositions [22]. The density and spin structure factor for the BEC-BCS transition was calculated [23]. Interferometric measurement schemes of the spatial pairing order have been proposed based on the atomcounting statistics in the output channels [24]. Pairing fluctuations of trapped Fermi gases have been studied in [25]. The number statistics of Fermi and Bose gases has also been investigated in $[26,27]$. In recent experiments [28] the spatial structure of an atomic cloud has been directly observed (without the expansion used in most other experiments). This makes it possible to determine the density fluctuations either by repeating the experiment many times or by taking densities at different positions in a homogeneous system to extract the statistics. Atomic shot noise has been experimentally investigated both in bosonic and fermionic systems [29-35].

In this article we propose to use the full counting statistics of density fluctuations as a tool to gain access to the manybody nature of the ground state of a fermionic cloud in the BEC-BCS crossover regime (for other work on full counting statistics in ultracold atomic gases see [36] on the experimental and [37-40] on the theoretical side). Our main result is a general expression for the particle number statistics of the mean-field BCS wave function. In the limiting cases, the statistics allows a straightforward interpretation. Deep in the molecular BEC limit the statistics is Poissonian-i.e., that of independent pairs of atoms. In the opposite limit, on the BCS side of the crossover, the fluctuations are strongly suppressed and reflect the particle-hole symmetry. The statistics in the crossover regime differs strongly from both the BCS and BEC limits and will be discussed based on several numerical results.

\section{COUNTING STATISTICS OF DENSITY FLUCTUATIONS}

We consider an atomic cloud with spatial distribution $n(x)$ and divide the system into bins; see Fig. 1. We are interested in the probability $P(N)=\langle\delta(N-\hat{N})\rangle$ to find $N$ atoms in a "bin" of the system; see Fig. 1. Here, $\hat{N}=\int_{V_{\text {bin }}} \hat{n}(x)$ is the bin number operator, $V_{\text {bin }}$ is the bin volume, and $\hat{n}(x)$ denotes the density operator. The bin volume is assumed to be much 

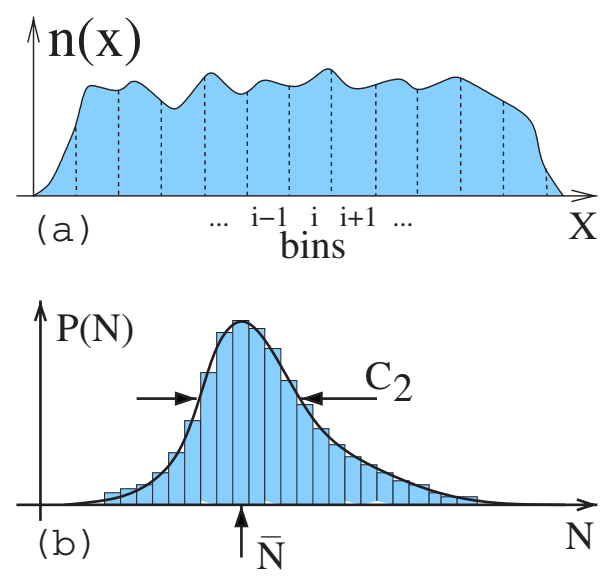

FIG. 1. (Color online) (a) Sketch of a typical atomic number density. The measured observable is the atom number $N$ given by $n(x)$ integrated over the bin volume (the bins are indicated by the dashed lines). (b) Histogram of probabilities to find $N$ particles in a bin.

smaller than the volume of the full system, but still much larger than the mean interparticle distance cubed. Hence, a single bin can be considered as a grand-canonical system with the surrounding atomic cloud serving as the reservoir.

In practice, it is more convenient to study the Fourier transform of $P(N)$, which is related to the cumulant generating function (CGF) $S(\chi)$ via

$$
e^{-S(\chi)}=\sum_{N} e^{i \chi N} P(N)=\left\langle e^{i \chi \hat{N}}\right\rangle .
$$

The cumulants $C_{n}$ are defined in a standard way as $S(\chi)=-\sum_{n} C_{n}(i \chi)^{n} / n !$ and can be used to characterize the full counting statistics (FCS). We recall that $C_{1}=\bar{N}$ is the average number of atoms in a bin and $C_{2}=\left\langle(N-\bar{N})^{2}\right\rangle$ measures the width of the number distribution. The third cumulant is proportional to the skewness and, therefore, a measure of the asymmetry of the distribution function. As we will see later, the third cumulant in a fermionic system is related to deviations from particle-hole symmetry.

\section{NONINTERACTING FERMIONS AND BOSONS}

We start by recalling some properties of noninteracting Fermi and Bose gases at a given temperature $T$. Here, individual atoms are independent and we can obtain the statistics as

$$
S(\chi)=\mp \sum_{k} \ln \left[1 \pm f_{ \pm}(k)\left(e^{i \chi}-1\right)\right],
$$

where $f_{ \pm}(k)=\left\{\exp \left[\left(\epsilon_{k}-\mu\right) / k_{B} T\right] \pm 1\right\}^{-1}$ is the Fermi (Bose) occupation function. The chemical potential is determined by the average atom number-i.e., $C_{1}=\bar{N}=\Sigma_{k} f_{ \pm}$. Obviously, the number statistics of fermions and bosons differs drastically in the degenerate regime. In the nondegenerate regime $f_{ \pm}$is small and we find the Poissonian statistics of classical particles $S(\chi)=-\bar{N}[\exp (i \chi)-1]$, for both fermions and bosons.
For degenerate fermions $k_{B} T \ll \epsilon_{F}$ the statistics is

$$
S(\chi)=-i \chi \bar{N}-\left(D k_{B} T / 4 \epsilon_{F}\right) \bar{N} \chi^{2} .
$$

Here and in the following, $S(\chi)$ is defined in the interval $[-\pi, \pi]$ and extended periodically. Thus, particle-number fluctuations are suppressed by $T / \epsilon_{F}$ in comparison to the classical case [41]. Remarkably, the statistics is Gaussian and consequently all cumulants $C_{n}$ for $n \geqslant 3$ vanish. This behavior resembles fermions in a one-dimensional (1D) wire [42] and can be interpreted as a consequence of antibunching. Note that the Gaussian nature of the statistics is a consequence of particle-hole symmetry and is therefore strictly limited to the degenerate regime. Higher-order corrections in $k_{B} T / \epsilon_{F}$ will introduce deviations from the Gaussian limit and lead, e.g., to the appearance of higher-order odd cumulants, which are directly related to deviations from the perfect particle-hole symmetry.

In contrast to that, we obtain quite a different behavior for free bosons. Approaching the degeneracy temperature $T_{C}^{\mathrm{BEC}}$ $=2 \pi \hbar^{2} n^{2 / 3} / m \zeta(3 / 2)^{2 / 3}$ [43] from above, the fluctuations are enhanced due to the large factor $f_{-}(k)\left(f_{-}(k)+1\right)$. In the condensed regime, the occupation of the ground state becomes macroscopically large and the grand-canonical approach is no longer valid $[44,45]$. This can be seen if we take the limit $T=0$ of Eq. (2), leading to $S(\chi)=\ln \left[1-\bar{N}\left(e^{i \chi}-1\right)\right]$, which corresponds to a negative binomial distribution, and the fluctuations therefore diverge according to $C_{n} \sim \bar{N}^{n}$. As in the grandcanonical ensemble the chemical potential is $\mu=0$ below the critical temperature $T<T_{C}^{\mathrm{BEC}}$, an arbitrarily large number of bosons can be transferred from the reservoir into the bin, leading to unphysically large fluctuations. In order to find the correct fluctuations in this case, we calculate the FCS from Eq. (1) explicitly. We divide the boson operator into $a_{k}=b_{k}$ $+c_{k}$, where

$$
b_{k}=\int_{V_{\text {bin }}} d^{3} r e^{i k r} \Psi(r), \quad c_{k}=\int_{V^{\prime}} d^{3} r e^{i k r} \Psi(r),
$$

where $V^{\prime}$ is the volume $V$ without the volume $V_{\text {bin }}$ of the bin. We consider a fully condensed state of $N_{\text {tot }}$ noninteracting bosons $|\psi\rangle=\left(a_{0}^{\dagger}\right)^{N_{\text {tot }} \mid}|\mathrm{vac}\rangle=\left(b_{0}^{\dagger}+c_{0}^{\dagger}\right)^{N_{\text {tot }}}|\mathrm{vac}\rangle$. Using Eq. (1) for the bin number operator $\hat{N}=\Sigma_{k} b_{k}^{\dagger} b_{k}$ we obtain a binomial statistics for a noninteracting bosonic gas in a bin at $T=0$ :

$$
S(\chi)=-N_{\text {tot }} \ln \left(1+\frac{V_{\text {bin }}}{V}\left(e^{i \chi}-1\right)\right) .
$$

For bin volumes $V_{\text {bin }} / V \ll 1$ the particle-number statistics becomes $\quad$ Poissonian-i.e., $\quad S(\chi) \approx-\bar{N}\left(e^{i \chi}-1\right), \quad$ where $\bar{N}=N_{\text {tot }} V_{\text {bin }} / V$.

\section{BCS GROUND STATE}

We now turn to a Fermi gas with an attractive interaction parameterized by a scattering length $a$. The Hamiltonian for the full system is number conserving and as such shows no number fluctuations at all. Here we consider a bin-i.e. a small subsystem-which we assume to be described by the 
non-number-conserving BCS Hamiltonian [7]. A straightforward ansatz for a non-number-conserving many-body state, which takes the pairing interaction into account, is the BCS wave function, which will be used in the following and is known to correctly describe both the BCS and BEC limits [8-10]. We will later show that the approach also reproduces the correct counting statistics in the two limits, and we therefore prefer to use this transparent, almost analytical approach instead of more complex canonical approaches. The BCS wave function is given by

$$
|\mathrm{BCS}\rangle=\prod_{k}\left(u_{k}+v_{k} c_{k \uparrow}^{\dagger} c_{-k \downarrow}^{\dagger}\right)|0\rangle .
$$

The variational procedure yields $v_{k}^{2}=1-u_{k}^{2}=\left[1-\left(\epsilon_{k}\right.\right.$ $\left.-\mu) / E_{k}\right] / 2$, where $E_{k}^{2}=\left(\epsilon_{k}-\mu\right)^{2}+\Delta^{2}$ is the energy of quasiparticle excitations. The order parameter $\Delta$ and the chemical potential are fixed by the self-consistency equations

$$
\Delta=-\lambda \sum_{k} u_{k} v_{k}, \quad \bar{N}=2 \sum_{k} v_{k}^{2}
$$

where $\lambda$ is the BCS coupling constant. After renormalization of the coupling constant $\lambda$ and considering only the lowenergy limit, the gap equation can be related to the twoparticle scattering amplitude $[9,46]$.

The product form of the BCS wave function greatly simplifies the calculation of the statistics, since different $k$ states can be treated separately. For a single pair of states $(k \uparrow,-k \downarrow)$ the sum over all possible configurations can be easily performed:

$$
e^{-S_{k}(\chi)}=\left\langle\mathrm{BCS}\left|e^{i\left(\hat{n}_{k \uparrow}+\hat{n}_{-k \downarrow}\right) \chi}\right| \mathrm{BCS}\right\rangle=u_{k}^{2}+v_{k}^{2} e^{2 i \chi} .
$$

The sum of all states yields the result

$$
S(\chi)=-\sum_{k} \ln \left[1+v_{k}^{2}\left(e^{2 i \chi}-1\right)\right] .
$$

This is one of the main results of our paper. It is valid in two and three dimensions; the dimension will only enter into the density of states when transforming the sum over $k$ into an energy integration via the standard expression $N_{D}$ $=m^{D / 2}(2 \epsilon)^{D / 2-1} / 2 \pi^{D-1} \hbar^{D}$ for $D=2,3$. It should be noted that in a strictly two-dimensional system the low-energy scattering amplitude vanishes $\sim-1 / \ln \epsilon$ and consequently the gap equation (7) shows a logarithmic divergence for $\epsilon \rightarrow 0$. However, for the more realistic situation of a quasi-twodimensional cloud (i.e., a three-dimensional trapped atomic cloud strongly confined in one dimension), this singularity is eliminated. Results derived for the strictly two-dimensional situation are still valid for the quasi-two-dimensional case, however with the chemical potential $\mu$ shifted by the groundstate energy.

We now discuss some limiting cases in which analytical expressions can be obtained. On the BEC side, $\mu<0$ and $\Delta \ll|\mu|$ leads to $v_{k}^{2} \ll 1$ for all energies and allows one to expand the logarithm in Eq. (9). The result is

$$
S(\chi)=-\frac{\bar{N}}{2}\left(e^{2 i \chi}-1\right)
$$

which corresponds to a Poissonian number statistics of pairs of atoms. This supports the picture of strongly bound pairs in a coherent state. Note that the factor of 2 in the exponent leads to exponentially growing cumulants in the fermion number-viz., $C_{n}=2^{n} \bar{N} / 2$. We therefore expect strong fluctuations. Remarkably, the result (10) is in accordance with the number statistics of condensed bosons in a bin with volume much less than the total volume; see the expression given after Eq. (5), which was derived using the canonical formalism. Since we are counting single fermions instead of bosons in the present case, the counting field $\chi$ is replaced by $2 \chi$ and there is a prefactor $1 / 2$. This agreement is quite remarkable, since the starting point of our approach is the grand-canonical formalism. It is an indication that using the BCS wave function to describe the number fluctuations of a small subsystem works surprisingly well.

On the BCS side the situation is quite different. Here $\mu$ $=\epsilon_{F} \gg \Delta$ and we obtain

$$
S(\chi)=-i \chi \bar{N}-\pi \bar{N} D \frac{\Delta}{4 \epsilon_{F}}[|\cos (\chi)|-1] .
$$

We observe that the first term is dominant but contributes only to the first cumulant. The fluctuations come from the second term in Eq. (11) which is smaller by a factor of $\Delta / \epsilon_{F}$. Furthermore, similar to the degenerate Fermi gas, the odd cumulants $C_{n}$ for $n \geqslant 3$ vanish, which is again a consequence of particle-hole symmetry.

Due to the constant density of states in 2D, we can obtain an analytical expression for the CGF for arbitrary $\mu$ and $\Delta$, which reads

$$
\begin{aligned}
S(\chi)= & -\bar{N} \frac{\Delta}{\epsilon_{F}}\left[\cos (\chi) \operatorname{atan}\left(\frac{2 \epsilon_{F}}{\Delta} e^{i \chi}\right)-\operatorname{atan}\left(\frac{2 \epsilon_{F}}{\Delta}\right)\right] \\
& -\frac{\bar{N}}{2} \frac{\mu}{\epsilon_{F}} \ln \left[1+v_{0}^{2}\left(e^{2 i \chi}-1\right)\right] .
\end{aligned}
$$

Here $v_{0}^{2}=\left(1+\mu / \sqrt{\mu^{2}+\Delta^{2}}\right) / 2$ is the BCS coherence function for $k=0$. While we do not have an analytical expression in $3 \mathrm{D}$, we expect a similar behavior as in $2 \mathrm{D}$. This will be corroborated later by comparing the numerically obtained cumulants in 3D to the 2D case.

In Fig. 2 we show how the density noise changes through the BEC-BCS crossover. Going from the BEC to the BCS regime strongly suppresses the fluctuations, in agreement with our previous discussion. The inset shows the fluctuations normalized to $\Delta / \epsilon_{F}$; they approach a constant value in the BCS limit. Another important piece of information gained from a noise measurement is the order parameter, which can be extracted from a measurement of $C_{2}$ in the BCS limit, Eq. (11), as $\Delta / \epsilon_{F}=4 C_{2} / \pi \bar{N} D$. Figure 3 shows the third cumulant. The global behavior is rather similar to the second cumulant; i.e., $C_{3}$ is strongly reduced going from the BEC limit to the BCS limit. However, the behavior of $C_{3}$ normalized to $\Delta / \epsilon_{F}$ shown in the inset is qualitatively different from that shown in the inset of Fig. 2 since $C_{3}$ vanishes also in the BCS limit. Note also that $C_{3}$ vanishes faster in the $2 \mathrm{D}$ case than in the $3 \mathrm{D}$ case. To understand the behavior of the third cumulant in more detail we recall that $C_{3}$ is related 


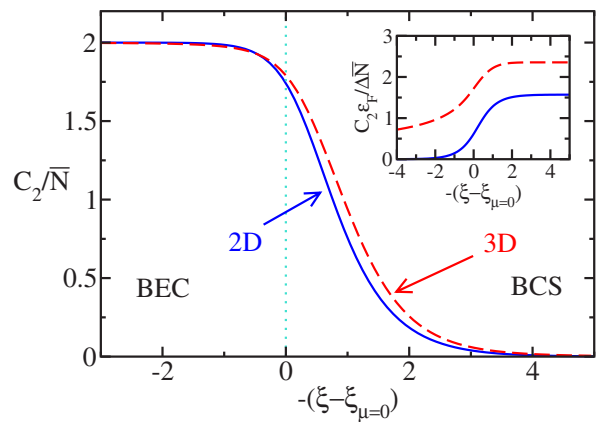

FIG. 2. (Color online) Second cumulant $C_{2}$ as a function of $\xi$ $=1 / k_{F} a$. For the strongly confined, quasi-two-dimensional system (2D), the Fermi vector can be approximated by the inverse of the ground-state size: $k_{F}=\pi / \ell_{0}$. The dotted line corresponds to $\xi_{\mu=0}$. The inset shows $C_{2}$ normalized to $\Delta / \epsilon_{F}$.

to the skewness of the distribution; i.e., $C_{3}$ is a measure of the difference between positive and negative fluctuations. To see this, we note that an elementary binomial event has the property $\ln \left\{1+v_{k}^{2}[\exp (i \chi)-1]\right\}=i \chi+\ln \left\{1+u_{k}^{2}[\exp (-i \chi)-1]\right\}$. Using this property and the particle-hole symmetry of $v_{k}^{2}$ in the BCS limit, we can rewrite the CGF in the BCS regime as $i \chi \bar{N}+N_{0} \int d \epsilon \ln \left\{1+v_{k}^{2} u_{k}^{2}[\cos (2 \chi)-1]\right\}$. Here we have used the fact that the density of states close to $\epsilon_{F}$ can be approximated by a constant $N_{0}$. The second term is even in $\chi$ and therefore only contributes to even cumulants, whereas all odd cumulants for $n \geqslant 3$ vanish. The difference between $2 \mathrm{D}$ and $3 \mathrm{D}$ seen in Fig. 3 is caused by the (small) energy dependence of the $3 \mathrm{D}$ density of states, which is absent in $2 \mathrm{D}$.

\section{FINITE TEMPERATURES}

We would now like to discuss the effect of finite temperatures in a qualitative way. The excitations in the BCS theory are fermionic quasiparticles. This is a good approximation on the BCS side of the transition. The fluctuations (measured by the second cumulant $C_{2}$ ) will be reduced with increasing temperature, since they approach the linearly increasing $C_{2}$ of the free Fermi gas that is lower at the critical temperature than $C_{2}$ at $T=0, C_{2}^{\mathrm{free}}\left(T_{C}^{\mathrm{BCS}}\right)<C_{2}(0)$. At even higher temperatures $T \geqslant T_{F}, C_{2}$ reaches the classical Poisson value $\bar{N}$. The situation on the BEC side of the crossover will be very different. According to our result the statistics is a Poissonian distribution of molecules and, hence, $C_{2}$ is doubled in comparison to the classical value for the atomic gas [47]. Upon increasing the temperature, the main effect on the fluctua-

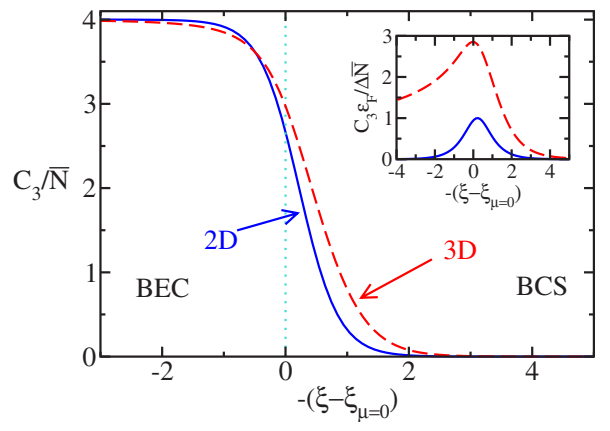

FIG. 3. (Color online) Third cumulant $C_{3}$ as a function of $\xi$ as defined in Fig. 2. The dotted line corresponds to $\xi_{\mu=0}$. The inset shows $C_{3}$ normalized to $\Delta / \epsilon_{F}$.

tions will be a breaking up of molecules, which will reduce the second cumulant $C_{2}$ above a dissociation temperature to finally reach the value for the classical gas. Thus, we expect quite a different temperature dependence of the number fluctuations on the BEC or BCS side of the transition.

\section{CONCLUSION}

In conclusion, we have calculated the full counting statistics of number densities in an ultracold fermionic atomic cloud with attractive interactions. The number statistics in the vicinity of the BEC-BCS crossover displays interesting features which reveal the nature of the many-body ground state. Poissonian fluctuations of a molecular condensate on the BEC side are strongly suppressed on the BCS side. The size of the fluctuations in the BCS limit is a direct measure of the pairing potential. We have also discussed the BECBCS crossover of the third cumulant and the temperature dependence of the second cumulant. These quantities can be accessed experimentally and provide additional information on the many-body ground state in the crossover regime. The concept of counting statistics in ultracold gases opens interesting possibilities to study the interplay between coherence and correlation in quantum many-body systems.

\section{ACKNOWLEDGMENTS}

We would like to thank A. Lamacraft for discussions. This work was financially supported by the Swiss NSF, the NCCR Nanoscience, and the European Science Foundation (QUDEDIS network). This research was supported in part by the National Science Foundation under Grant No. NSF PHY0551164.
[1] M. H. Anderson, J. R. Ensher, M. R. Matthews, C. E. Wieman, and E. A. Cornell, Science 269, 198 (1995); K. B. Davis, M.-O. Mewes, M. R. Andrews, N. J. van Druten, D. S. Durfee, D. M. Kurn, and W. Ketterle, Phys. Rev. Lett. 75, 3969 (1995).

[2] B. DeMarco and D. S. Jin, Science 285, 1703 (1999).
[3] A. G. Truscott, K. E. Strecker, W. I. McAlexander, G. B. Partridge, and R. G. Hulet, Science 291, 2570 (2001).

[4] F. Schreck, L. Khaykovich, K. L. Corwin, G. Ferrari, T. Bourdel, J. Cubizolles, and C. Salomon, Phys. Rev. Lett. 87, 080403 (2001).

[5] S. R. Granade, M. E. Gehm, K. M. O’Hara, and J. E. Thomas, 
Phys. Rev. Lett. 88, 120405 (2002).

[6] K. M. O'Hara, S. L. Hemmer, M. E. Gehm, S. R. Granade, and J. E. Thomas, Science 298, 2179 (2002).

[7] J. Bardeen, L. N. Cooper, and J. R. Schrieffer, Phys. Rev. 108, 1175 (1957).

[8] D. M. Eagles, Phys. Rev. 186, 456 (1969).

[9] A. J. Leggett, in Modern Trends in the Theory of Condensed Matter, edited by A. Pedalski and J. Przystawa (Springer, Berlin, 1980).

[10] P. Nozières and S. Schmitt-Rink, J. Low Temp. Phys. 59, 195 (1985).

[11] S. Simonucci, P. Pieri, and G. C. Strinati, Europhys. Lett. 69, 713 (2005).

[12] R. Grimm, e-print arXiv:cond-mat/0703091.

[13] T. Bourdel, J. Cubizolles, L. Khaykovich, K. M. F. Magalhães, S. J. J. M. F. Kokkelmans, G. V. Shlyapnikov, and C. Salomon, Phys. Rev. Lett. 91, 020402 (2003).

[14] M. Bartenstein, A. Altmeyer, S. Riedl, S. Jochim, C. Chin, J. H. Denschlag, and R. Grimm, Phys. Rev. Lett. 92, 203201 (2004).

[15] J. Kinast, S. L. Hemmer, M. E. Gehm, A. Turlapov, and J. E. Thomas, Phys. Rev. Lett. 92, 150402 (2004).

[16] C. A. Regal, M. Greiner, and D. S. Jin, Phys. Rev. Lett. 92, 040403 (2004).

[17] M. W. Zwierlein, C. A. Stan, C. H. Schunck, S. M. F. Raupach, A. J. Kerman, and W. Ketterle, Phys. Rev. Lett. 92, 120403 (2004).

[18] M. Greiner, C. A. Regal, C. Ticknor, J. L. Bohn, and D. S. Jin, Phys. Rev. Lett. 92, 150405 (2004).

[19] C. Chin, M. Bartenstein, A. Altmeyer, S. Riedl, S. Jochim, J. H. Denschlag, and R. Grimm, Science 305, 1128 (2004).

[20] E. Altman, E. Demler, and M. D. Lukin, Phys. Rev. A 70, 013603 (2004).

[21] A. Lamacraft, Phys. Rev. A 73, 011602(R) (2006).

[22] R. Bach and K. Rzazewski, Phys. Rev. Lett. 92, 200401 (2004).

[23] H. P. Büchler, P. Zoller, and W. Zwerger, Phys. Rev. Lett. 93, 080401 (2004).

[24] I. Carusotto and Y. Castin, Phys. Rev. Lett. 94, 223202 (2005).

[25] L. Viverit, G. M. Bruun, A. Minguzzi, and R. Fazio, Phys. Rev. Lett. 93, 110406 (2004).

[26] M. Budde and K. Mølmer, Phys. Rev. A 70, 053618 (2004).

[27] D. Meiser and P. Meystre, Phys. Rev. Lett. 94, 093001 (2005).
[28] M. Bartenstein, A. Altmeyer, S. Riedl, S. Jochim, C. Chin, J. H. Denschlag, and R. Grimm, Phys. Rev. Lett. 92, 120401 (2004).

[29] S. Fölling, F. Gerbier, A. Widera, O. Mandel, T. Gericke, and I. Bloch, Nature (London) 434, 481 (2005).

[30] M. Schellekens, R. Hoppeler, A. Perrin, J. Viana Gomes, D. Boiron, A. Aspect, and C. I. Westbrook, Science 310, 648 (2005).

[31] M. Greiner, C. A. Regal, J. T. Stewart, and D. S. Jin, Phys. Rev. Lett. 94, 110401 (2005).

[32] T. Rom, Th. Best, D. van Oosten, U. Schneider, S. Fölling, B. Paredes, and I. Bloch, Nature (London) 444, 733 (2006).

[33] J. Esteve, J.-B. Trebbia, T. Schumm, A. Aspect, C. I. Westbrook, and I. Bouchoule, Phys. Rev. Lett. 96, 130403 (2006).

[34] T. Jeltes, J. M. McNamara, W. Hogervorst, W. Vassen, V. Krachmalnicoff, M. Schellekens, A. Perrin, H. Chang, D. Boiron, A. Aspect, and C. I. Westbrook, Nature (London) 445, 402 (2007).

[35] I. B. Spielman, W. D. Phillips, and J. V. Porto, Phys. Rev. Lett. 98, 080404 (2007).

[36] A. Öttl, S. Ritter, M. Köhl, and T. Esslinger, Phys. Rev. Lett. 95, 090404 (2005).

[37] A. Lamacraft, e-print arXiv:cond-mat/0512580.

[38] A. Nunnenkamp, D. Meiser, and P. Meystre, New J. Phys. 8, 88 (2006).

[39] A. Kuklov and H. Moritz, e-print arXiv:cond-mat/0609531.

[40] B. Capogrosso-Sansone, E. Kozik, N. Prokof'ev, and B. Svistunov, Phys. Rev. A 75, 013619 (2007).

[41] Y. Castin, e-print arXiv:cond-mat/0612613.

[42] L. S. Levitov, H. Lee, and G. B. Lesovik, J. Math. Phys. 37, 4845 (1996).

[43] The expression for $T_{C}^{\mathrm{BEC}}$ is calculated for a three-dimensional gas confined by rigid walls. $\zeta$ is the Riemann zeta function.

[44] L. D. Landau, and E. M. Lifshitz, Statistical Physics, 3rd ed. (Butterworth-Heinemann, Oxford, 1984), Pt. I, Sec. 113.

[45] A. J. Leggett, Quantum Liquids (Oxford University Press, Oxford, 2006).

[46] M. Randeria, J.-M. Duan, and L. Y. Shieh, Phys. Rev. B 41, 327 (1990).

[47] Note that here the fluctuations of atoms are discussed. This should not be confused with the fluctuations of the molecule number. 